\title{
Corrigendum: Machine Learning- Based Analysis of Magnetic Resonance Radiomics for the Classification of Gliosarcoma and Glioblastoma
}

\author{
Zenghui Qian ${ }^{1+}$, Lingling Zhang ${ }^{2+}$, Jie Hu ${ }^{1}$, Shuguang Chen ${ }^{3}$, Hongyan Chen ${ }^{2}$, \\ Huicong Shen ${ }^{2}$, Fei Zheng ${ }^{2}$, Yuying Zang ${ }^{2}$ and Xuzhu Chen ${ }^{2 *}$ \\ ${ }^{1}$ Department of Neurosurgery, Beijing Tiantan Hospital, Capital Medical University, Beijing, China, ${ }^{2}$ Department of Radiology, \\ Beijing Tiantan Hospital, Capital Medical University, Beijing, China, ${ }^{3}$ School of Mathematical Sciences, Nankai University, \\ Tianjin, China
}

OPEN ACCESS

Approved by:

Frontiers Editorial Office,

Frontiers Media SA, Switzerland

*Correspondence:

Xuzhu Chen

radiology888@aliyun.com

${ }^{\dagger}$ These authors share first authorship

Specialty section: This article was submitted to

Cancer Imaging and

Image-directed Interventions,

a section of the journal

Frontiers in Oncology

Received: 11 September 2021 Accepted: 04 October 2021 Published: 18 October 2021

Citation:

Qian Z, Zhang L, Hu J, Chen S, Chen $H$, Shen $H$, Zheng $F$, Zang $Y$ and Chen $X$ (2021) Corrigendum: Machine

Learning-Based Analysis of Magnetic Resonance Radiomics

for the Classification of Gliosarcoma and Glioblastoma.

Front. Oncol. 11:774369. doi: 10.3389/fonc.2021.774369
Keywords: gliosarcoma, glioblastoma, machine learning, radiomics, differentiation

\section{A Corrigendum on}

Machine Learning-Based Analysis of Magnetic Resonance Radiomics for the Classification of Gliosarcoma and Glioblastoma

By Qian Z, Zhang L, Hu J, Chen S, Chen H, Shen H, Zheng F, Zang Y and Chen X (2021). Front. Oncol. 11:699789. doi: 10.3389/fonc.2021.699789

In the published article, there was a mistake in the order of authors. The co-first author was incorrectly written as the second author. The correct author list with the correct author order appears below.

“Zenghui Qian ${ }^{1 \dagger}$, Lingling Zhang ${ }^{2 \dagger}$, Jie Hu ${ }^{1}$, Shuguang $\mathrm{Chen}^{3}$, Hongyan $\mathrm{Chen}^{2}$, Huicong Shen ${ }^{2}$, Fei Zheng ${ }^{2}$, Yuying Zang ${ }^{2}$, Xuzhu Chen ${ }^{2 * »}$

The authors apologize for this error and state that this does not change the scientific conclusions of the article in any way. The original article has been updated.

Publisher's Note: All claims expressed in this article are solely those of the authors and do not necessarily represent those of their affiliated organizations, or those of the publisher, the editors and the reviewers. Any product that may be evaluated in this article, or claim that may be made by its manufacturer, is not guaranteed or endorsed by the publisher.

Copyright $(2021$ Qian, Zhang, Hu, Chen, Chen, Shen, Zheng, Zang and Chen. This is an open-access article distributed under the terms of the Creative Commons Attribution License (CC BY). The use, distribution or reproduction in other forums is permitted, provided the original author(s) and the copyright owner(s) are credited and that the original publication in this journal is cited, in accordance with accepted academic practice. No use, distribution or reproduction is permitted which does not comply with these terms. 